\title{
Drones-as-a-service: A management architecture to provide mission planning, resource brokerage and operation support for fleets of drones
}

\author{
Juan A. Besada, Ana M. Bernardos, Luca Bergesio, Diego Vaquero, Iván Campaña, José R. Casar
}

\begin{abstract}
Drone operators, institutions (e.g. law enforcement, rescue agencies, preservation organisms, etc.) and companies (logistics, surveillance, inspection, etc.), are constituting themselves as drone operators, at the same time that increase the size of their fleets of drones. Nowadays, each operator independently manages its drones, on service request. Beyond this isolated approach, many operation scenarios would be much better addressed if automated means for coordinated resource allocation and simultaneous drone control were available. In this paper, we describe a microservice-oriented architecture that provides organization and optimization functionalities to orchestrate drone operation. The platform built on the proposed architecture may be used to enable a single operator to optimize the management of its fleet of drones, but also to facilitate coordination of different resources towards a common goal, assuming a drone-as-a-service operation mode. The architecture is validated through a specific use case, for real time allocation of drone resources to enable fast emergency response.
\end{abstract}

Keywords-drone, fleet management, emergency management, unmanned traffic management, optimization, drone-as-a-service

\section{INTRODUCTION}

Nowadays, it is already feasible to find in press and literature numerous service situations in which drones are being used. It is the case of industry infrastructure inspection (oil refineries, telecom towers, wind turbines) or to patrol pipelines, electricity power lines or railways. Mapping and surveying of mining, construction sites, border areas (e.g. maritime surveillance), agriculture fields (to analyze crop health), fisheries or forestry are also common applications. In emergency response, drones are starting to be explored both for prevention (of earthquakes, floods, hurricanes or fires) and intervention. In this second case, to deliver emergency medical supplies (e.g. defibrillators for cardiac arrest) or to transfer real time images to fire-fighters, police, etc. Another example of use is the use of drones for communications, as HALE (High Altitude Long Endurance) or/and HAPS (High Altitude Pseudo Satellite).

Thus drones-based applications are already wide-ranging, and according to the European Drones Outlook Study [1], the market of the drones will explode in the next thirty years: some 7 million consumer leisure drones are expected to be operating across Europe and a fleet of 400.000 is expected to be used for commercial and government missions in 2050. More versatile platforms, enhanced sensors, autonomous flight, consolidated UTM (Unmanned Traffic Management) systems to enable coordination with traditional Air Traffic Management, authorization and control support-tools, indoor operations, etc., are some of the topics in the research, policy and industry agendas.

Among the mentioned multiple challenges that need to be overcome to make drones-based services part of our daily life (in particular in cities), there is also a need for tools enabling better coordination and management of the available drones, this said in particular for drone operators governing mid-sized fleets or for a set of drone operators that may offer coordinated service to a third party, following a drone-as-a-service approach. Nowadays, in the public sector, institutions and state services are making grow their own fleets, in an independent and non-coordinated manner: the final user detects a need that may be better addressed through the use of drones, and the institution/stakeholder decides to acquire one or several units, organize personnel search or training and, finally becomes a drone operator with the purpose of flying its drones when needed (which may be necessary in every day or scattered operations). It will be solely the public user that will determine how and when to fly the drones. In practice, this means for example that in an emergency scenario, urban police, firefighters, or army emergency services will have drones ready to fly, but the coordination among the available drones needs to be handle at upper-organization level, thus being less immediate and cost-efficient, and undoubtfully more complicated.

Even whether this approach is not efficient, it is still sustainable, but as fleets are forecasted to increase, the possibility of sharing resources to e.g. provide support to multiple urban services by a centralized fleet (thus optimizing acquisition, renewal and maintenance) or to put resources from different public owners together to optimize their use should be possible. With this approach in mind, this paper describes an architecture designed with the purpose of facilitating easy mission definition and the use of drones-as-a-service, i.e. optimal drone and resource allocation and planning. The objective behind the architecture is to efficiently manage heterogenous fleets of drones, from different owners and pilots. The proposed architecture is thought to facilitate the management of the whole workflow: before, during and post flight. Previously to the flight, the mission definer may easily describe the mission to accomplish (in terms of location and schedule, but also in terms of sensing needs, fly modes manual or automated - and pilots and platforms), search for available materials and human resources to complete the 
operation, identify the best of options and generate the flight plan for the pilot to inject it into the chosen Ground Control System. The architecture is also designed to enable real-time retrieval of video streaming and telemetry from flying drones, which is represented in different control interfaces for operation monitoring. Historical logs are available after flight, together with the recorded operation.

With this context in mind, the paper is structured as follows. Section II briefly review current research on experiences to manage drone operations. Section III introduces the service scenario and main system requirements. Section IV describes the proposed architecture and Section V details some of the human-computer interfaces that have been deployed over it to use the tool to coordinate resource use for emergency support. Finally, Section VI concludes the work.

\section{RELATED WORK}

How to provide control tools for non-expert users to specify drone-based missions is nowadays a challenge. For example, authors in [2] propose a system in which a detailed flight plan is automatically generated for each multi-copter involved, preventing collisions between multi-copters and obstacles, and ensuring the preservation of no-fly zones. How to facilitate the teleoperator task is addressed in [3]; in this system, a specific interface provides real-time environment-adaptive viewpoints that are automatically configured to improve safety and operation. This enables to handle situations in which nearby objects can generate collision hazards and frequent occlusion can hinder accurate manipulation. The system uses simultaneous localization and mapping based reconstruction (known as SLAM) and combines robot position and orientation, and information of 3D point-cloud to modify the user viewpoint to maximize visibility. In [4], a tool to define and real-time handle inspection missions is described. Through the use of simple geometric volumes, missions can be easily configured, and the real-time workings of the system enables on-the-fly mission tailoring and adjustment if there is a need for modification.

Determining optimal observation altitudes is the main issue addressed in [5]; authors aim at minimizing the cost of the flight while ensuring the surveillance of all target. In [6], a fleet of autonomous lightweight UAVs coordinate themselves real time. The use of UAV fleets for atmospheric science is addressed in [7]; how to manage the trade-offs between payload, endurance or ease of deployments are some of the aspects that are considered by the SkyScanner project, which aims at developing a fleet of UAV to sample cumuli. To do so, it is needed optimal flight control in intelligent cooperative missions.

Safety and security of autonomous drone missions during the flight is the issue in [8]. Authors describe a tool that can be used for formally verifying these aspects by using the continuous video streaming forwarded to a control element to drive the flight. Linked to security, an increasing interest has been driven towards the definition of adequate humancomputer interfaces both for Ground Control Systems and others, as accidents involving drones due to operators' errors are somehow frequent. For example, [9] reports a study meant to design GCS interfaces for multiple RPAS control, with the objective of enhancing operators' performance.
The proposal in [10] describes a platform that offers an abstraction layer between the end-user and the drone, with the objective of automating the drone flight requirements, i.e. performing high-level control operations for the drone and integrating these workings in an event-driven control platform to achieve multi-drone automated control without compromising performance. Real-time information (telemetry) is also delivered.

Prospective studies such as [11] focus on demonstrating the economic potential of multi-sided platforms for remote operation of drones over the internet, i.e. the way a dronesharing platform (as the proposed architecture) can provide technology solutions such as remote internet-based operation and advanced autonomy. Additionally, this work also evaluates restrictions on remote operation and regulation needs to create spaces for innovation and competitive advantage.

\section{MAIN SYSTEM REQUIREMENTS}

\section{A. Service scenario}

The architecture to be designed needs to take into consideration all the main stakeholders who can intervene in a mission with drones. A mission is the definition of the route to be followed by the drone, composed by: a purpose goal (e.g. a set of inspections), a flight plan (a number of waypoints and drone dynamics) and a predicted trajectory to be flown. Unmanned Aerial Systems (UAS) are composed by different subsystems, in particular i) the drone of Unmanned Aerial Vehicle (UAV), which can be RPAS (remote piloted) or autonomous, and its airborne component (payload), ii) the Ground Control System/Station (GCS), iii) the Ground control link and iv) other components, depending on the applications.

The main roles to be implemented by the stakeholders in the service field considered as relevant to are the following:

- Mission definer/creator: it will be the entity in charge of dealing with mission definition. In practice, it can be a user willing to provide a given service (e.g. emergency assessment and response, packet delivery, surveillance, etc.) by using her own drone fleet or available resources from different drone operators.

- Mission Supervisor: Person who visualizes mission results and provides the pilot with feedback. It may be the same person who creates the mission.

- Mission Viewer: Person who visualizes video/data captured by the drone. It may be the same person who creates the mission.

- Pilots: pilots are in charge of setting up and flying drones, both in manual or automated modes. Each pilot may have a set of drones owned/allocated or be available to fly any drone authorized in her license.

- Payload Manager: Person in charge of setting up and controlling the payload. the missions will have specific sensing and operating needs. For example, a mission can rely on real-time video streaming, while other may only need thermal camera feedback or be designed to deliver a given good in a target area. The drones and payloads needed to fulfil each mission differ, thus it is necessary to manage these resources to check availability. 
- System Manager: Person who overviews the system behavior: drone fleet, assignments, ongoing missions...

- Logistics personnel: they may be needed to transport drones and payloads to specific locations to make service feasible.

With these seven roles in mind, we have divided a dronebased mission into four macro-steps: design, preparation, execution and post-execution.

During design, a drone operator or the person in charge defines a mission through a tailored human-computer interface (which will be further described in Section V). The HMI must be agile and easy to understand, thus making smooth for the user design a mission under certain circumstances (e.g. medical emergency or a wildfire). Missions may be composed of several flights, so the HMI helps the mission creator to optimize the resource allocation among the flights. Flights are composed of a take-off geo-reference, an operation zone and a landing point. Resources belong to an operator, but in some cases, the concept needs to support resource sharing (e.g. the police and the fire brigade during a fire). The resources are: pilots, logistics personnel, drones, batteries, payloads and sensors/actuators (parts mounted on a payload). The system calculates the fastest option to have a drone with the needed equipment flying over the operation zone.

Pilots and logistics personnel are assumed to carry a mobile device that will guide them during the preparation of the mission: the pilot and the ready-to-fly drone have to be at the takeoff point together at a specific time. The system must consider the initial position of the resources involved, the traffic, the time needed to collect the parts and to assemble the drone and the weather forecast. Moreover, a pilot may even be a logistics personnel, thus the system must also consider this option during the resource optimization.

Once the pilot and the drone are ready to take off, the execution step begins. In order to reduce the time to reach the operation zone, the system estimates the fastest trajectory considering the drone model, its total weight, the elevation and the shape of the ground and the wind. It also generates two flight plans, one for the takeoff point - operation zone and one for the operation zone - landing point. These flight plans are in a format understandable by the drone (e.g. MAVlink), so they can be flown automatically. In particular, MAVLink is a very lightweight, header-only message marshalling library for micro air vehicles, which has extensively been tested e.g. on PX4, PIXHAWK, APM and Parrot AR platforms. During the flight, the drone sends its telemetry and the data collected by its sensors to the system. Different HMIs permit to see the mission from different places and by different users (e.g. by a mission supervisor from a control center or by a policeman near the operation zone using a smartphone). Data from the drone may also be processed in real-time adding some specific module to the system (e.g. video analytics to detect people using a thermal camera stream in a search and rescue operation).

After the mission, all data are stored in a central server and they can be accessed during a period of time by the users who have the permission. Sensitive data are deleted after a certain period of time following some content policies created to respect privacy rules (e.g. videos are deleted after two months unless an institution requests to maintain them during a longer period).

\section{B. Functional / non-functional requirements}

Considering the procedures and features reported in the previous section, we can extrapolate the functional and nonfunctional requirements of the system. First the functional requirements are listed:

- It may be used to create fast missions, so it must have a user-friendly interface that permits to define a mission in the simplest way possible.

- Drone-as-a-service workings: Resources should be shared among different operators in some critical cases.

- Decision Support: The system must select the best resources, among the available ones, for a mission, considering the time to reach the operation zone as the most important parameter for the optimization algorithm. Some of the best solutions ( 3 or 5 ) must be shown to the user for the final decision.

- The optimization algorithm must consider the position and availability of the resources, the shape of the ground, the fastest routes, the traffic forecast, the weather forecast and the performance of the drone(s).

- The takeoff point must be calculated by the system in order to minimize the time to arrive to the operation zone, considering all the parameters of the previous requirement.

- The system must generate two flight plans in the droneunderstandable language to give the possibility to fly automatically from the take-off point to the operation zone and from the operation zone to the landing point.

- The system must guide the logistics personnel and the pilots, instructing them about the steps to do to prepare the drone for the mission, about the mission and about how to reach the takeoff point. The instructions must be provided through a smartphone application using notification in real-time.

- Resources should be tracked at all times to know their position. The smartphone application for pilots and logistics personnel should permits to track users and equipments.

- During the flights, the system must be able to receive, process and store the information from all the sensors mounted on the drones.

- This information may be shown in real-time to different users, on different devices and it must be associated to the user permissions.

- All gathered data must be stored and deleted according to the local privacy law.

The non-functional requirements that the system must accomplish are the following.

- Usability. All HMIs must be user-friendly and they must be able to provide information in real-time to permit to perform and to follow a mission

- Reliability and Availability. The system must create periodical backups of all the databases involved and it must have a very high rate of availability. 


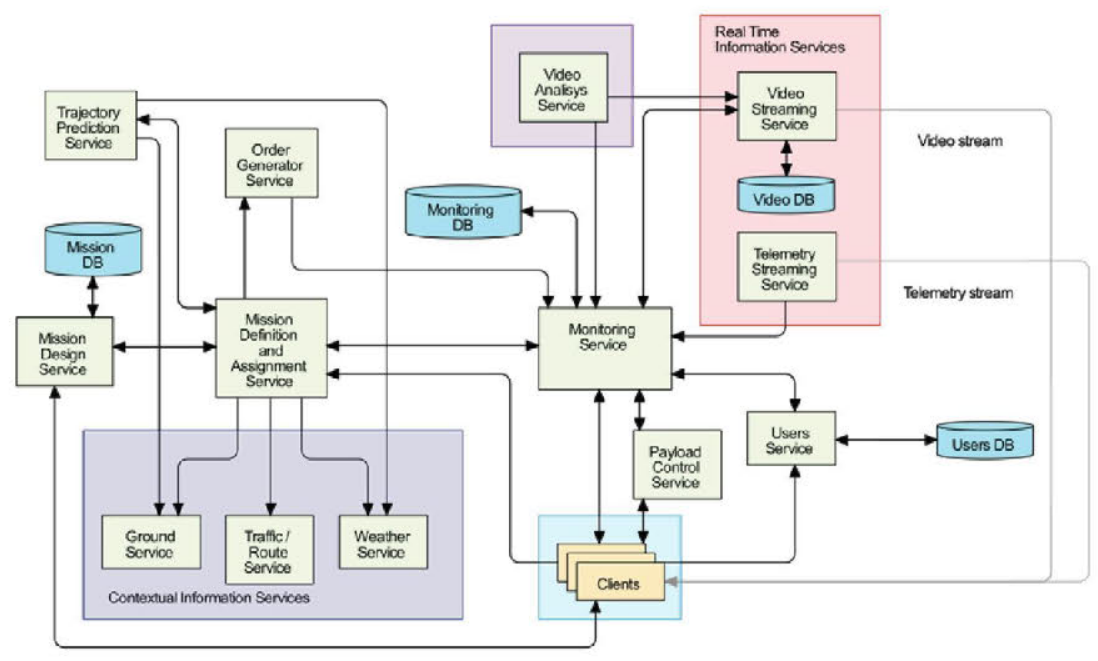

Fig. 1. System architecture.

- Performance. The system could be used for emergencies, so it must be responsive and fast, and it should use the least amount of resources possible (e.g. CPU, RAM).

- Supportability. It must be easy to modify/upgrade some of the existing components and to include new ones.

- Replication. It must be reusable and re-instantiated for different end-clients (e.g. municipalites).

\section{MICROSERVICE-ORIENTED ARCHITECTURE}

This Section details the approach used to design proposed microservice-oriented architecture, which need to accomplish all the functional and non-functional requirements detailed in Section III. The architecture is based on microservices for role separation and scalability. The microservices provide a RESTful API that permits the interaction between a client and the platform itself. An MQTT broker dispatches the messages for the real time communications. Both the RESTful API and the MQTT messages use the JSON format for the data. The architecture is based on microservices because this way it is possible to easily separate the role of each component simplifying the development and the deployment of the system. It also permits to include new components to extend the capabilities of the platform and it is a good solution for scalability. The components that consume the data provided by the platform are the clients. There is a different client for each possible role of the user.

Fig. 1 depicts the architecture schema. It has two main services which communicate with the other components to provide all the procedures and features. They are the Monitoring Service and the Mission Definition and Assignment Service (MDA). The first one maintains the state and the updated position of all the resources that the system must handle. The MDA use this information, combined with the ground shape, the traffic/route information and the weather forecast, to assign the resources to a mission created by a user. Ground, Traffic/Route and Weather services are context services: they gather the information from external services (e.g. Google Maps, Bing, OpenWeather, etc.) and they perform some initial calculations on the data before passing them to the MDA.

The Trajectory Prediction Service also uses the processed data provided by the Ground and the Weather services. The prediction of the trajectory is important to decide the takeoff point which is calculated by the MDA mashing up context information and the available resources, not by the user who creates the mission.

The creation of a mission is done by a client with the support of the Mission Design Service, which helps the user to define a mission applying filters on the available resources and suggesting some optimal options. Once a mission is created, it is stored in a specific database waiting for the execution, which may even occur immediately. The mission is stored with all the needed resources, with the set of instructions to prepare the drone for the logistics personnel and with the trajectory described in a drone-understandable language.

When it is the time to begin a mission, the Order Generator Service uses the set of instructions and the current position of the needed resources to guide, from one side, the logistics personnel to assemble and carry the drone to the takeoff point, and from the other side, the pilot to the same point.

The Monitoring Service continuously tracks as many resources as possible, using the GPS installed on some devices when they are on (typically drones and some sensors) or the information provided by the logistics personnel and by the pilot using a smartphone application (e.g. batteries transported by a logistics personnel are tracked assigning the same position of that user). All this information is stored in a database controlled by the Monitoring Service.

During the flight, the telemetry sent by the drone is also used to track the drone, thus its position is continuously updated in the monitoring database, while all the telemetry is stored in the mission database, associated with the correct mission. In this manner, it will be possible to review the entire mission after it is over. 


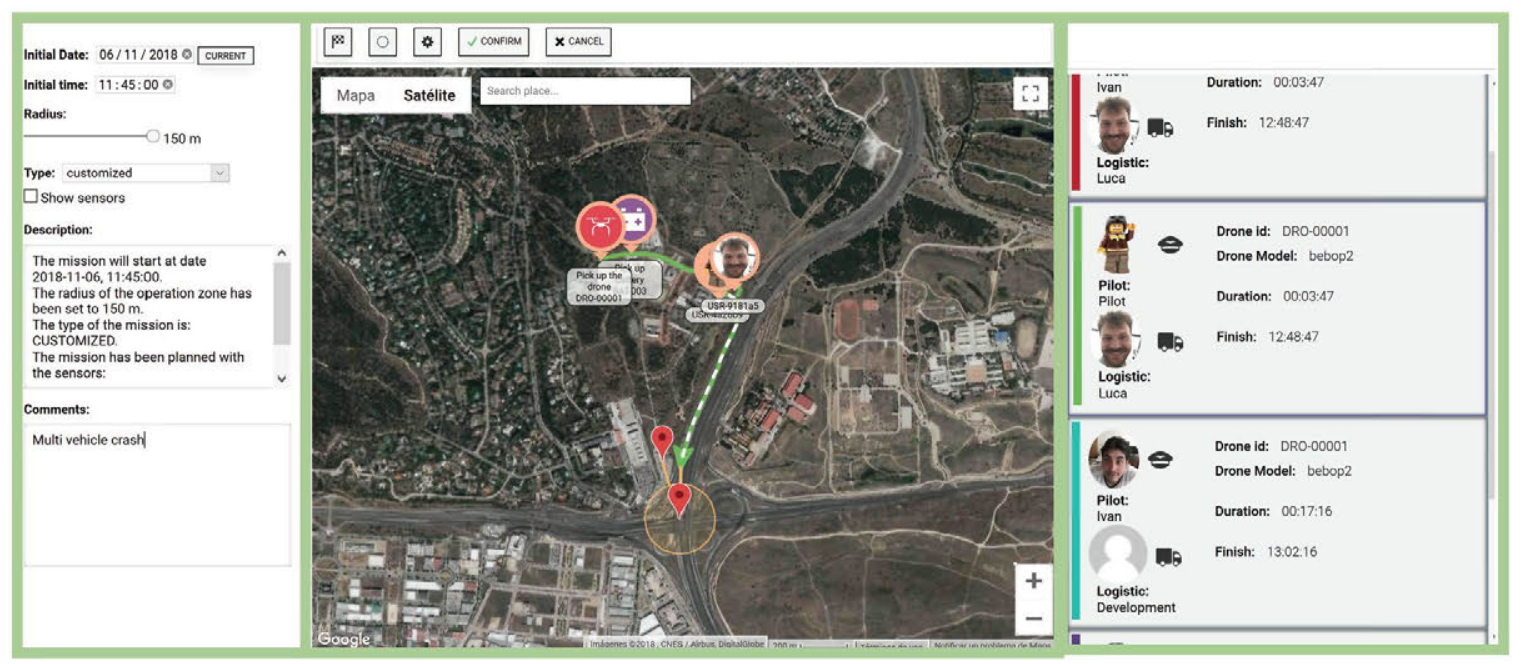

The services in the group of Real Time Information Services are the interfaces between the drone and the Monitoring Service and they are active only when the drone is flying. Each service works with a specific type of data, the system includes by default a service for the telemetry and another for the video sources (two typical data provided by most commercial drones), but it is possible to include additional services to treat data from other sensors (e.g. gas, thermal, LIDAR, etc.). Other microservices may be added to the system to perform some specific operation on the real-time data provided by the sensors mounted on the drone. Since we can work with video streams, we added a service (Video Analytics Service) to automatically recognize people or objects in a real-time video. Video Streaming Service has a dedicated database to save all the videos. MDA controls the time and decides when a video must be deleted according to some internal policies. A Payload Control Service permits to interact with the payload and the sensors mounted on the drone remotely (when available and controllable), for example to take a picture or to adjust the zoom of the camera.

Users Service handles the permission to access the resources and to see the mission before, during and after the execution. It also permits to create the specific configuration where two or more operators can share their resources for a particular mission. This service has its own database to store all the information about the users and their permissions.

Additional microservices can be easily added to the system to add new features and several instances of each service may be executed to increase the general performances of the architecture.

\section{VALIDATION THROUGH USE CASE: ALLOCATING RESOURCES FOR EMERGENCY RESPONSE}

This section describes a use case of the proposed architecture for real time allocation of drone resources in emergency scenarios. In such situations, the response speed is critical since there may be people in danger.
Our system enables fast emergency response and it facilitates the fleet management. To illustrate the capabilities of the system we propose a multiple crash-landing scenario, in which aerial images are required. Once the crash has been detected, the emergency system receives a call, which is attended by the mission controller. Using the provided information of the emergency (i.e. location and description) $\mathrm{s} /$ he creates a mission using our platform. Each mission is defined by the following elements:

- The sensors or actuators to be used during the mission. The tool offers the possibility to select one predefined mission type with a set of associated sensors (e.g. "Toxic Analysis" or "Recognition") or to manually specify the elements to be used.

- The location and radius of the operation zone in which the manual flight will be performed, in this case the emergency location. The radius is used to provide the human pilot a theoretical dimension of the area that must be flown over.

- The landing point for the drone. This location specifies where the drone must land once the mission has been carried out.

The radius of the operation zone and the landing point are merely informative, given that once in the area, it may be required to leave it or to land in a different position (e.g. the pilot decides to extend the flight time in the area, hence the drone may not be capable to reach the defined landing point).

Once defined the mission, the system calculates a set of possible options to perform it. For each option, the system selects a pilot, a drone and a logistic person (The person in charge of gathering all the required resources and take them near the operation area, where he will meet the pilot) considering the available resources. Additionally, the system generates two flight plans, one from the estimated takeoff point (which is calculated considering the actual position of the pilot) to the operation zone and one from the operation zone to the landing point. These flight plans are generated in drone- 
specific format (e.g. MAVLink), and they can be used by the pilot in the first and last stage of the flight.

To conclude the mission generation, the creator selects one of the available options either using the fastest suggested option or any other one. The mission is then completed, and all the resources are blocked and assigned. At the same time, notifications are sent to the pilot and logistic, with the relevant information for each one. Fig. 2 shows a screenshot of the tool during the mission creation process. Fig. 2 is divided into three vertical sections. In section A, the mission controller introduces the mission information (operation zone radius and sensors / mission type). Operation zone and landing position are introduced using the map in $B$. Once the calculations are done, the tools show the possible options in $\mathrm{C}$, whose details can be seen in the map.
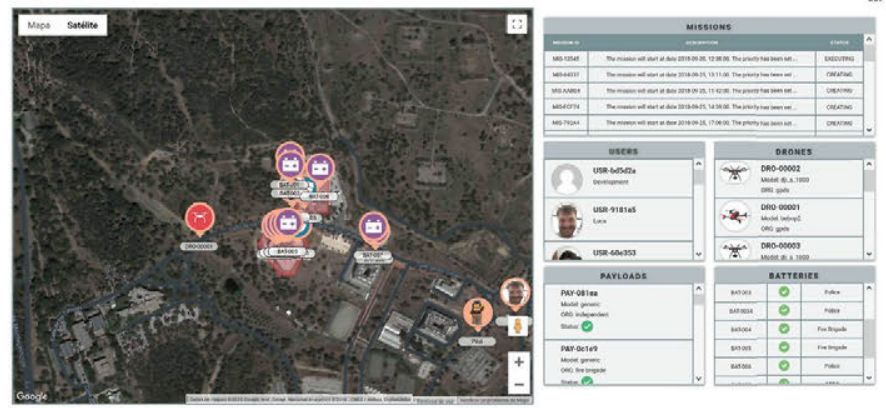

Fig. 3. Resource planner (II).

From the HMI, the mission controller may control the mission in real time by receiving real time video and positions from the pilot, the logistic person and the drone. The system also offers the possibility to inspect past missions, offering support to clarify past issues.

Using the platform, the system controller can also locate every resource on the system and every stakeholder in real time, in addition to the missions that are currently being carried out. Fig. 3 shows a screenshot of this functionality. In the left side of the figure a map appears, in which all elements are represented. On the right side elements, the user can select any of the elements to obtain further details. Fig 4. Shows the HMI both in a Tablet and a videowall (which simulates a multiviewer control interface).

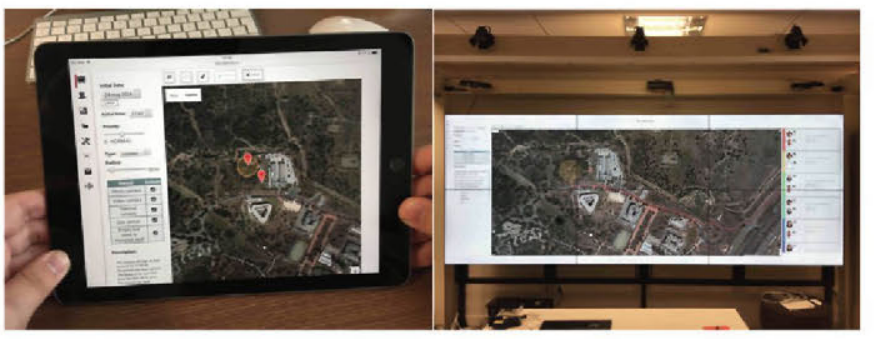

Fig. 4. a) Mission definition on a tablet and b) Tracking missions execution in a control wall.

\section{CONCLUSIONS AND FURTHER WORK}

Public services (police, fire brigades, maintenance service providers, etc.) are starting to use or have revealed their interest towards drone operations. Each stakeholder is providing its own solution for operation but shared and coordinated resources is necessary to fully exploit the possibilities. With that objective in mind, this paper presents a drone-as-a service enabling architecture and its practical implementation, which has been customized to provide fast-response in emergency environment. The platform enables dynamic mission creation, resources assignment and flight monitoring and posterior exploitation, and can be used to manage a owned fleet of drones or to act as service broker for different drone operators in a coordinated manner.

We consider that these types of platforms are necessary to evolve the drone economy. Nowadays, the most frequent mode operation mode includes enterprise drones, which are individual. Some applications, such as collaborative emergency response or urban logistics will require to manage BVLOS semi-autonomous drone fleets. The concept of drones-as-aservice connects the general public to end services offered by autonomous drones, in a future no pilot will be required, and the proposed architecture also envisions this future.

\section{ACKNOWLEDGMENT}

This work was supported in part by EIT Digital through the project drones112, Universidad Politécnica de Madrid Project RP1509550C02 and the Spanish Ministry of Economy and Competitiveness under Grant TEC2017-88048-C2-1-R.

\section{REFERENCES}

[1] SESAR (2016). European Drones Outlook Study. Unlocking the value for Europe.

[2] Bozhinoski, D., Di Ruscio, D., Malavolta, I., Pelliccione, P., \& Tivoli, M. (2015, November). Flyaq: Enabling non-expert users to specify and generate missions of autonomous multicopters. In Automated Software Engineering 30th IEEE/ACM Intl. Conference on (pp. 801-806). IEEE.

[3] Thomason, J., Ratsamee, P., Kiyokawa, K., Kriangkomol, P., Orlosky, J., Mashita, T., ... \& Takemura, H. (2017, March). Adaptive View Management for Drone Teleoperation in Complex 3D Structures. In Proc. Intl. Conf. on Intelligent User Interfaces (pp. 419-426). ACM.

[4] Besada, J. A., Bergesio, L., Campaña, I., Vaquero-Melchor, D., LópezAraquistain, J., Bernardos, A. M., \& Casar, J. R. (2018). Drone Mission Definition and Implementation for Automated Infrastructure Inspection Using Airborne Sensors. Sensors, 18(4), 1170.

[5] Zorbas, D., Pugliese, L. D. P., Razafindralambo, T., \& Guerriero, F. (2016). Optimal drone placement and cost-efficient target coverage. Journal of Network and Computer Applications, 75, 16-31.

[6] Bevacqua, G., Cacace, J., Finzi, A., \& Lippiello, V. (2015, January). Mixed-Initiative Planning and Execution for Multiple Drones in Search and Rescue Missions. In ICAPS (pp. 315-323).

[7] Lacroix, S., Roberts, G., Benard, E., Bronz, M., Burnet, F., Bouhoubeiny, E., ... \& Renzaglia, A. (2016, April). Fleets of enduring drones to probe atmospheric phenomena with clouds. In EGU General Assembly Conference Abstracts (Vol. 18, p. 12445).

[8] Apvrille, L., Roudier, Y., \& Tanzi, T. J. (2015, May). Autonomous drones for disasters management: Safety and security verifications. In Radio Science Conference, 1st URSI Atlantic (pp. 1-2). IEEE.

[9] Luongo, S., Di Gregorio, M., Vitiello, G., \& Vozella, A. (2018, October). Human Machine Interface Issues for Drone Fleet Management. In Intl. Conference on Human Systems Engineering and Design: Future Trends and Applications (pp. 791-796). Springer, Cham.

[10] Areias, B., Humberto, N., Guardalben, L., Fernandes, J. M., \& Sargento, S. (2018). Towards an Automated Flying Drones Platform. In VEHITS (pp. 529-536).

[11] Chornenky, D. (2018). A multi-sided platform for remote operation of shared drones: deriving strategic opportunities from regulatory trends (Doctoral dissertation, Massachusetts Institute of Technology). 